Article

\title{
Experimental Study on the Impact Characteristics of Cavitation Bubble Collapse on a Wall
}

\author{
Jing Luo, Weilin $\mathrm{Xu}$ *, Jun Deng, Yanwei Zhai and Qi Zhang \\ State Key Laboratory of Hydraulics and Mountain River Engineering, Sichuan University, \\ Chengdu 610065, China; luojing@scu.edu.cn (J.L.); djhao2002@scu.edu.cn (J.D.); \\ zaiyanwei0422@163.com (Y.Z.); scu_zq@163.com (Q.Z.) \\ * Correspondence: xuwl@scu.edu.cn; Tel.: +86-028-8540-1301
}

Received: 20 August 2018; Accepted: 14 September 2018; Published: 15 September 2018

check for updates

\begin{abstract}
As a hydrodynamic phenomenon, cavitation is a main concern in many industries such as water conservancy, the chemical industry and medical care. There are many studies on the generation, development and collapse of cavitation bubbles, but there are few studies on the variation of the cyclic impact strength on walls from the collapse of cavitation bubbles. In this paper, a high-speed dynamic acquisition and analysis system and a pressure measuring system are combined to study the impact of a cavitation bubble generated near a wall for various distances between the cavitation bubble and the wall. The results show that (1) with the discriminating criteria of the impact pressure borne by the wall, the critical conditions for the generation of a micro-jet in the collapse process of the cavitation bubbles are obtained, and therefore collapses of cavitation bubbles near the wall are mainly divided into primary impact area collapses, secondary impact area collapses and slow release area collapses; (2) it can be seen from the impact strength of the cavitation bubble collapse on the wall surface that the impact of cavitation bubbles on the wall surface during the first collapse decreases as $\gamma$ (the dimensionless distance between the cavitation bubble and the wall) increases, but the impact of the second collapse on the wall surface increases first and then decreases sharply. When $\gamma$ is less than 1.33, the impact on the wall surface is mainly from the first collapse. When $\gamma$ is between 1.33 and 2.37, the impact on the wall surface is mainly from the second collapse. These conclusions have potential theoretical value for the utilization or prevention and control technologies for cavitation erosion.
\end{abstract}

Keywords: cavitation bubble; high-speed photography; impact pressure; micro-jet

\section{Introduction}

When the collapse of cavitation bubbles occurs near a wall, the wall will bear the cyclic impact from the collapse of the cavitation bubble. The characteristic of the interaction between the cavitation bubble and the wall has become the focus of many technical fields, such as water conservancy, shipbuilding, the chemical industry and many other industries.

Theoretical study about cavitation bubbles can be tracked back to the last century [1-3]. The study of the cavitation field mainly focuses on the damage and destruction of a solid wall surface upon cavitation bubble collapse [4,5].

Shaw et al. [6] used a laser to generate a cavitation bubble and combined this with Schlieren photography to conduct a study on the impact performance of the impact wave and micro-jet generated during the first collapse of a cavitation bubble on a wall. Adopting high-speed photographic equipment with a camera speed of up to 100 million frames per second to study the evolution properties of cavitation bubbles around a wall, Lindau and Lauterborn [7] discovered the annular form during the collapse of a cavitation bubble, the formation of a contrajet and the shock wave. Liu et al. [8] used the method of laser-inducing a cavitation bubble to conduct a study on the impacts of a micro-jet caused 
by laser-induced cavitation bubble collapse on a metallic copper surface, and found that the liquid-jet was the main damage mechanism in cavitation erosion. Xu et al. [9] studied the cavitation bubble dynamic characteristics on an aluminum surface by using the technology of a sensitive fiber-optic sensor based on the optical beam deflection principle. Ren et al. [10] used a hydrophone to conduct a study on the strength mechanism of impact from cavitation collapse within the range of the relative bubble-wall dimensionless distance $\gamma\left(\gamma=h / R_{\max }, R_{\max }\right.$ is the maximal radius of the cavitation bubble, $h$ is the distance between the center of the cavitation bubble and the wall) which is between 0.5 and 2.5. The above studies on the collapse of cavitation bubbles either focus on the first collapse or adopt the indirect method of hydrophone measurement. In addition, from the research on the impact of the micro-jet or high-velocity droplet on a wall with the use of high-speed photography, only qualitative rules can be obtained. However, measuring the microscopical area of the wall under the impact of the micro-jet or high-velocity droplet with the pressure test system provides a new quantitative method for solving millimeter-sized impact-related issues [11].

In recent years, some new research directions on cavitation have been proposed, such as extracorporeal shock-wave lithotripsy [12] and ultrasonic cleaning [13,14]. In addition, studies on the interaction between cavitation bubbles and cavitation bubbles, cavitation bubbles and air bubbles, cavitation bubbles and particles, and cavitation bubbles and ice blocks have also been reported. Fong et al. [15] adopted the underwater discharge technique to induce multiple cavitation bubbles so as to study the coupling effect between two cavitation bubbles generated at different instants. Pain et al. [16] studied the jet flow in an air bubble induced by a nearby cavitation bubble and discovered that the speed of such a jet flow can be up to $250 \mathrm{~m} / \mathrm{s}$. Luo et al. $[17,18]$ used the method of high-voltage discharge technology to generate a cavitation bubble to study the interaction between a cavitation bubble and an air bubble, and the interaction between two cavitation bubbles and a rigid wall. Goh et al. [19] studied the interactions between cavitation bubbles horizontally placed below the underwater slab and air bubbles attached to it and discovered that the ratio between the oscillation time of the air bubbles and the oscillation time of the cavitation bubbles is the important parameter impacting the micro-jet caused by cavitation bubble collapse. The interaction between a cavitation bubble and a particle was researched by adopting a high-voltage discharge technology to induce a cavitation bubble [20] and low-voltage underwater discharge technology to induce a cavitation bubble [21]. The interaction between ice and cavitation bubbles was researched by Cui et al. [22], and the direction of the micro-jet and the propagation of shock waves were captured.

Due to the very small volume and short life cycle of cavitation bubbles during the evolution, great difficulties have been imposed on related studies. Therefore, numerical simulation studies have been performed by various researchers. Coupled boundary element and finite element methods have been performed on bubble-structure interactions [23-25]. The boundary element method is used for research on the toroidal configuration and jet impact characteristics in the later stage of the collapse of cavitation bubbles in the inviscid and irrotational field [26-28] numerically simulated the late rebound phenomena of a cavitation bubble by the method of vortex lines. Instead of using a vortex surface, Klaseboer et al. [29] simulated the ring stage of a cavitation bubble with a vortex ring, and profoundly understood the dynamic characteristics of a cavitation bubble and the interaction with walls for different distances between the bubble and the wall, which was in agreement with the experimental results.

For the past few decades, many researchers have tried to find out the impulses associated with cavitation bubble collapse. In most existing studies, these results are only qualitative due to the non-uniform spatial sensitivity of the transducer. Therefore, in this paper, cavitation bubbles are induced by adopting a low voltage spark-discharge system, the cavitation bubble evolution and corresponding wall pressure change processes are measured simultaneously by the piezoresistive pressure sensor. The impact strength of the first collapse and rebound-regeneration collapse of the cavitation bubble on the wall surface is mainly analyzed with the high-speed photographic image and 
pressure testing data of the wall surface, and the influence of the dimensionless distance of the bubble wall on the impact strength of the wall surface is revealed.

\section{Experimental Setups}

\subsection{Cavitation Bubble Induction Device}

In order to get the impact characteristics of the cavitation bubble collapse on the wall, the experimental setup requires a cavitation bubble induction device, a high-speed dynamic collection and analysis system and a transient pressure testing system, as shown in Figure 1.

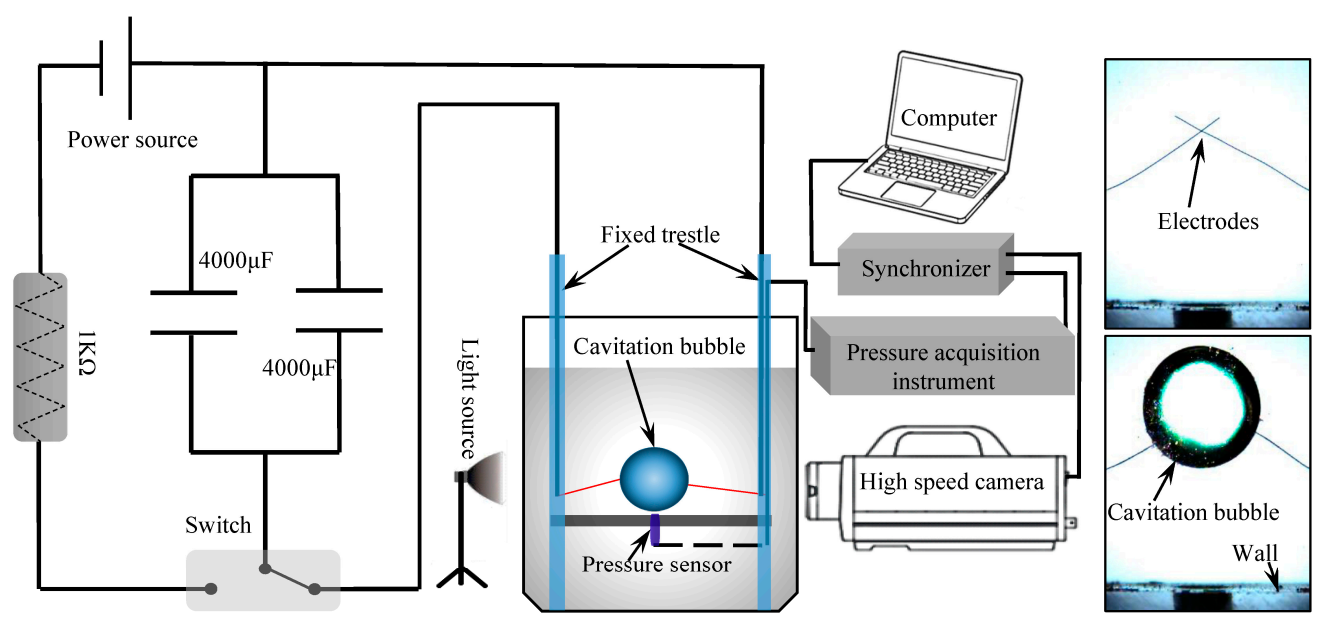

Figure 1. Schematic diagram of experimental facilities.

There are three ways to induce cavitation bubbles in quiescent water: by focusing a laser, by a high-frequency ultrasound wave or by an underwater discharge. The laser technique to induce a cavitation bubble has many advantages, such as its higher accuracy in controlling the focal spot, but the focusing method of the laser is rather complex and costly [30]. In the current work, the method of low-voltage underwater discharge was adopted. The method can accurately control the position and size of the cavitation bubble. To minimize possible interference, the diameter of the electrodes used is $0.15 \mathrm{~mm}$, and the bubbles created have a maximum radius of 30 times the diameter of the electrodes. The copper electrodes (length $50 \mathrm{~mm}$ ) are fixed in the trestles. The trestles are placed in a water tank $(40 \mathrm{~cm} \times 30 \mathrm{~cm} \times 40 \mathrm{~cm}$ ), which is filled with twice deionized water (at a constant temperature of $22^{\circ} \mathrm{C}$ ). To create the bubble, two capacitors are fully charged to the specified voltage (the adjustable range is $0 \mathrm{~V} \sim 100 \mathrm{~V}$ ) through a $1 \mathrm{~K} \Omega$ resistor, then switched to the discharge circuit, and the capacitor is discharged through the pair of crossed electrodes.

The life cycle of cavitation bubbles is very short; in order to study the impact process of the collapse of the cavitation bubble on the wall surface, a high-speed dynamic collection and analysis system and a transient pressure testing system must be adopted for synchronized recording. The high-speed dynamic collection and analysis system is composed of a high-speed camera, lens assembly, lighting equipment, etc., and the transient pressure testing system is composed of the resistance-type pressure sensor and the data acquisition instrument. These two systems are respectively connected to a computer through a synchronous trigger device. The Fastcam SA-Z high-speed camera (maximum acquisition rate: 1,000,000 fps, Photron Inc., Tokyo, Japan,) is used. The radius is of millimeter-size when the cavitation bubble generated after the discharge between electrodes in the water body evolves to its maximum volume. Thus, in order to obtain the clear outline of the cavitation bubbles, a micro-lens and LED lamp $(150 \mathrm{~W})$ must be adopted in the acquisition system and synchronized with the high-speed photography system. A framing rate of 150,000 frames per second is used to capture the bubble dynamics in this paper, with a shutter speed of $5.06 \mu$ s. The width of the frame is 256 pixels. 


\subsection{Measurement of Cavitation Bubbles During the Evolution Process and the Impact Process}

Considering the size of the cavitation bubbles (the radius of cavitation bubbles produced from the system in the experiment ranges from $5.00 \mathrm{~mm}$ to $12.00 \mathrm{~mm}$ ) and the time for the impact of the collapse of the bubble on the wall surface being very short, the size of the sensor in the transient pressure testing system must be small enough, and the sampling frequency and accuracy must be high enough; in addition, by observing the high-speed photographic images, the time for the impact of the micro-jet on the wall due to the collapse of the cavitation bubbles induced by the experimental system lasted about $100 \mu \mathrm{s}$. The minimum rise time was at least $10 \mu \mathrm{s}$. The pressure sensor used in the experimental system has a high-frequency bandwidth $(200 \mathrm{kHz})$. Therefore, with the existing sensor technology, the piezoresistive pressure sensor used in this paper can meet the requirements for the measurement of the impact of the collapse of cavitation bubbles on the wall surface. The radius of the sensing area of the sensor adopted in the test is $1.50 \mathrm{~mm}$ (the maximum measuring range is $40 \mathrm{MPa}$ ). The sampling frequency is $2 \mathrm{MHz}$, and the accuracy is $0.5 \%$ of the maximum measuring range.

The method of image gray processing is used to obtain the maximum radius of the cavitation bubble. In this paper, a circle radius with the same number of the pixels is regarded as the equivalent radius $R_{e q}$ of the cavitation bubble ( $R_{\max }$ is $R_{e q}$ when the cavitation bubble radius reaches maximum), and the centroid of the cavitation bubble in the high-speed photographic image is regarded as the center of the cavitation bubble. The dimensionless distance $\gamma$ is the ratio of the distance $h$ between the centers of the cavitation bubble and pressure sensor and the maximum value of the maximal equivalent radius $R_{\max }$ of the cavitation bubble. Figure 2 shows the data processing process: therein, the scatter diagram shows the relationship between the first cycle of the cavitation bubble time and the corresponding $R_{\max }$, and it can be seen that this data processing method provides good reproducibility.

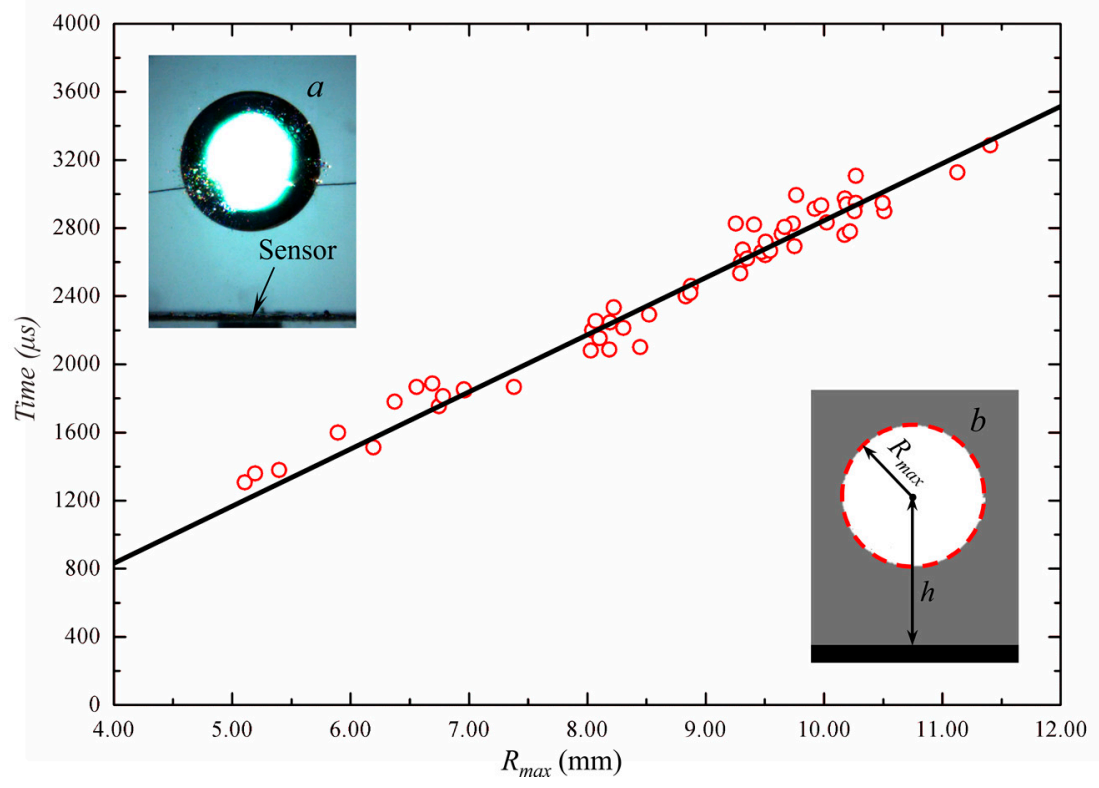

Figure 2. Processing method of characteristic parameters. (a) Cavitation bubble photograph; (b) characteristic parameters.

\section{Results and Discussion}

\subsection{Influence of Distance Between the Cavitation Bubble and Wall on the Development of Micro-Jetting}

A selected set of high-speed photographic images of the cavitation bubbles in the collapse stage for different $\gamma$ (dimensionless distances between the cavitation bubble and the wall) are shown in Figure 3. Therein, the wall surface is located at the bottom of the image. In the three sets of tests, the dimensionless distances $\gamma \mathrm{s}$ are $2.91,1.91$ and 0.83 , respectively, the maximum values $R_{\max }$ of the 
equivalent radius $R_{e q}$ are $9.40 \mathrm{~mm}, 9.31 \mathrm{~mm}$ and $9.47 \mathrm{~mm}$ respectively, and the distances $h$ from the center of the cavitation bubbles to the wall's surface when the cavitation bubbles expand to their maximum radii are $27.35 \mathrm{~mm}, 17.76 \mathrm{~mm}$ and $7.86 \mathrm{~mm}$, respectively.

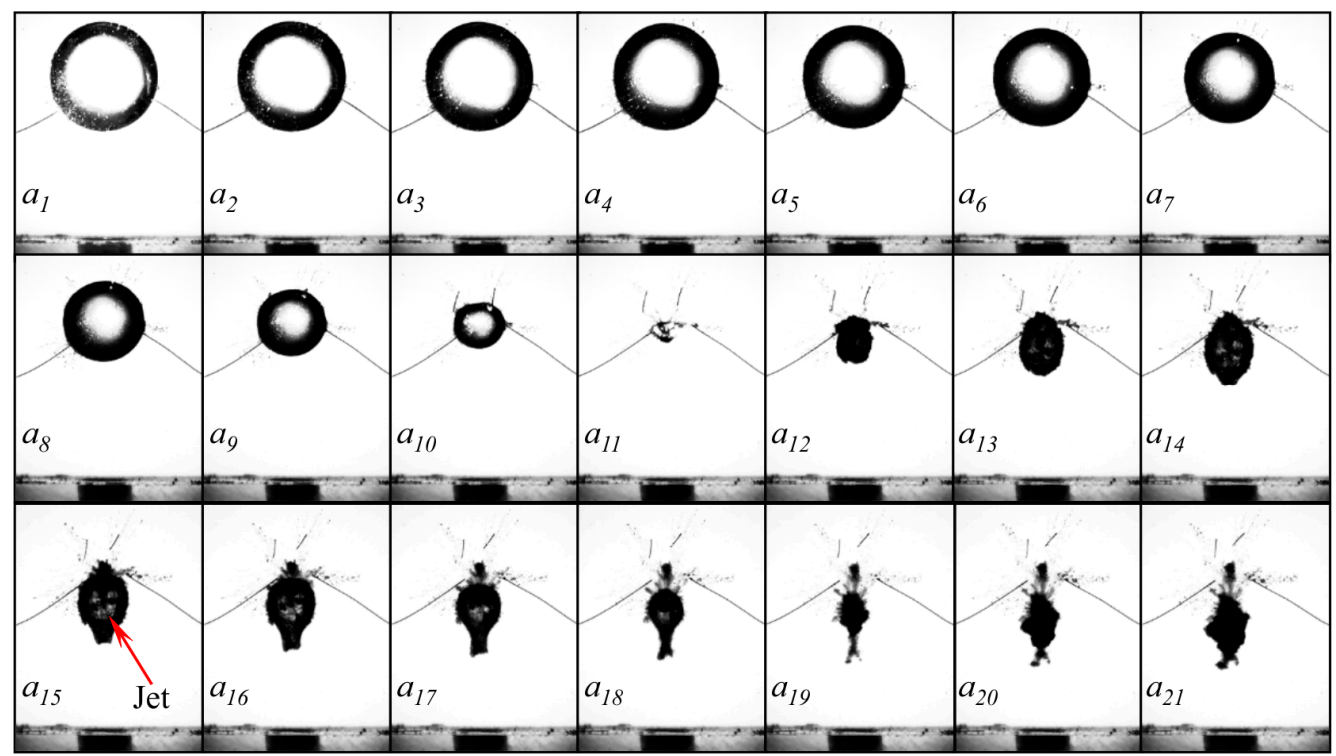

(a) $R=9.40 \mathrm{~mm} ; \gamma=2.91$

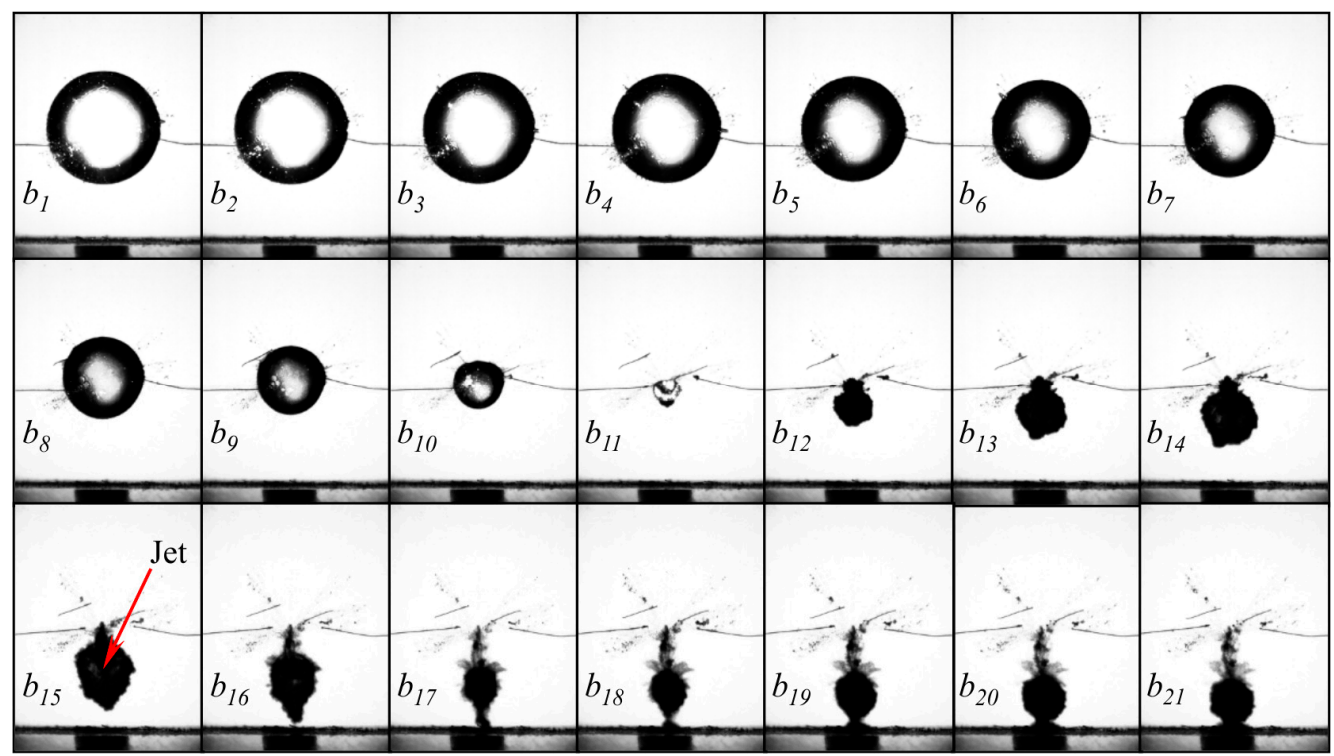

(b) $R_{\max }=9.31 \mathrm{~mm} ; \gamma=1.91$

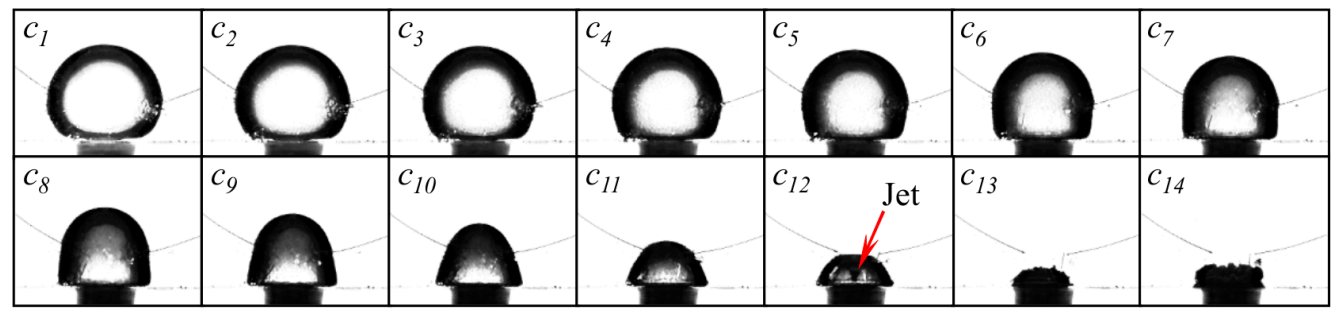

(c) $R_{\max }=9.47 \mathrm{~mm} ; \gamma=0.83$

Figure 3. High-speed photographic images of the cavitation bubbles in the contraction collapse stage under the conditions of different distances $\gamma$ (frame-rate: 150,000 fps; exposure time: $5.06 \mu$ s; frame width: $32.00 \mathrm{~mm}$ ). 
When the cavitation bubble expands to the maximum radius in the testing, as shown in Figure 3 $\left(a_{1}, b_{1}, c_{1}\right)$, the cavitation bubbles begin the contraction stage through an action of the external water body. With the gradual contraction of the cavitation bubble, due to the existence of the wall under the cavitation bubble, the surrounding water begins to fill the surrounding space released by the cavitation bubbles in their contraction process, and the upper and lower surfaces of the cavitation bubble begin to form asymmetrical shapes gradually. The wall surface under the cavitation bubble blocks the filling of water, resulting in a slow contraction speed of the lower surface of the cavitation bubble, while the upper surface belongs to the unbounded domain and contracts quickly. Thus, this asymmetric contraction gradually begins to form from the upper surface (as shown in $a_{9}, b_{9}$ and $c_{11}$ in Figure 3). The development of the asymmetric collapse of the surface has been accompanied by the shrinkage of cavitation bubbles to the minimum volume (as shown in $\mathrm{a}_{11}, \mathrm{~b}_{11}$ and $\mathrm{c}_{13}$ in Figure 3 ). The cavitation bubbles will rebound-regenerate after they shrink to the minimum volume, and as shown in Figure 3, the surface of the rebound cavitation bubble is not very smooth (as shown in $a_{12}$ and $b_{12}$ in Figure 3), but just as in the first cycle, the cavitation bubble will again undergo the expansion-contraction process. In the second expansion-contraction process, the surface is not smooth enough, but this non-smoothness is not enough to change the collapse direction of cavitation bubbles. In Figure $3 b$, the rebound cavitation bubbles move quickly toward the wall surface in the expansion-collapse process and impact onto the wall in the second collapse.

From the above three tests, the following can be concluded: when the dimensionless distance $\gamma$ is small, the micro-jet directly impacting the wall surface can be formed during the first asymmetric contraction of the cavitation bubble; as the dimensionless distance $\gamma$ has been increasing continuously, the cavitation bubbles will move to the wall surface quickly and will eventually impact on the wall surface. With the further increase of $\gamma$, cavitation bubbles do not impact on the wall surface through two or three times of contraction. Therefore, the collapse of cavitation bubbles near the wall surface can be divided into main impact area collapse, secondary impact area collapse and slow release area collapse as per the parameters of the distance between the cavitation bubble and wall.

\subsection{Impact of Cavitation Bubble Collapse on Wall}

The collapse position of cavitation bubbles near the wall surface can be obtained directly through high-speed photography for the development of the micro-jet under the above different $\gamma$ conditions, but the impact strength of cavitation bubbles on the wall surface during the first collapse, whether the impact can reach the wall surface during the second collapse, and the impact strength of the second collapse on the wall surface cannot be ascertained. With these questions, the high-speed dynamic collection and analysis system and transient pressure testing system are combined in this part, obtaining the impact process of the first and second cavitation bubble collapses on the wall surface (that is, the main impact area and secondary impact area). Figure 4 shows the impact process of the two groups of cavitation bubble collapses on the wall surface when the dimensionless distances $\gamma$ are 0.91 and 1.79 respectively. The wall surface is located at the bottom of the image, and the pressure sensor is placed inside of the wall surface.

The maximum radius of the cavitation bubble in Figure $4 \mathrm{a}$ is $9.98 \mathrm{~mm}$, and when the cavitation bubble reaches the maximum volume, the distance from the center to the wall surface is $9.08 \mathrm{~mm}$. When the electric spark discharges into the water during the induction of the cavitation bubble, the pressure value appears to fluctuate slightly, and the pressure returns to a normal condition after discharge. The subsequently generated cavitation bubbles will begin the expansion-contraction-collapse-rebound stage. In the cavitation bubble development process, the pressure on the wall surface is stable; when the cavitation bubble contracts to the minimum volume (the first expansion-contraction cycle is $2.91 \mathrm{~ms}$ ), the pressure on the wall surface sharply increases, abd from the contents of the previous section it can be seen that because of the short dimensionless distance $\gamma$, the collapse generated due to asymmetrical cavitation bubble contraction directly forms the micro-jet impacting the wall surface (shown as the image in Figure 4a), and the maximum peak 
value of impact pressure of the first cavitation bubble collapse on the wall surface is 19.37 MPa. After that, the peak pressure gradually decreases when the cavitation bubble is in the rebound-regeneration stage, the pressure on the wall surface is relatively stable for the whole rebound regeneration stage, and the pressure borne on the wall surface greatly increases again when the cavitation bubble contracts to the minimum volume again. The time period of the whole regeneration rebound is $1.23 \mathrm{~ms}$; the wall surface bears the collapse impact of the rebound cavitation bubble again, and by this time, the maximum pressure on the wall surface is $6.50 \mathrm{MPa}$. It can be seen from the whole impact process of the cavitation bubbles on the wall surfaces in the condition of $\gamma=0.91$ that the strength of the two impacts of cavitation bubbles on the wall surfaces will gradually reduce from $19.37 \mathrm{MPa}$ in the first impact to $6.50 \mathrm{MPa}$ in the second impact.

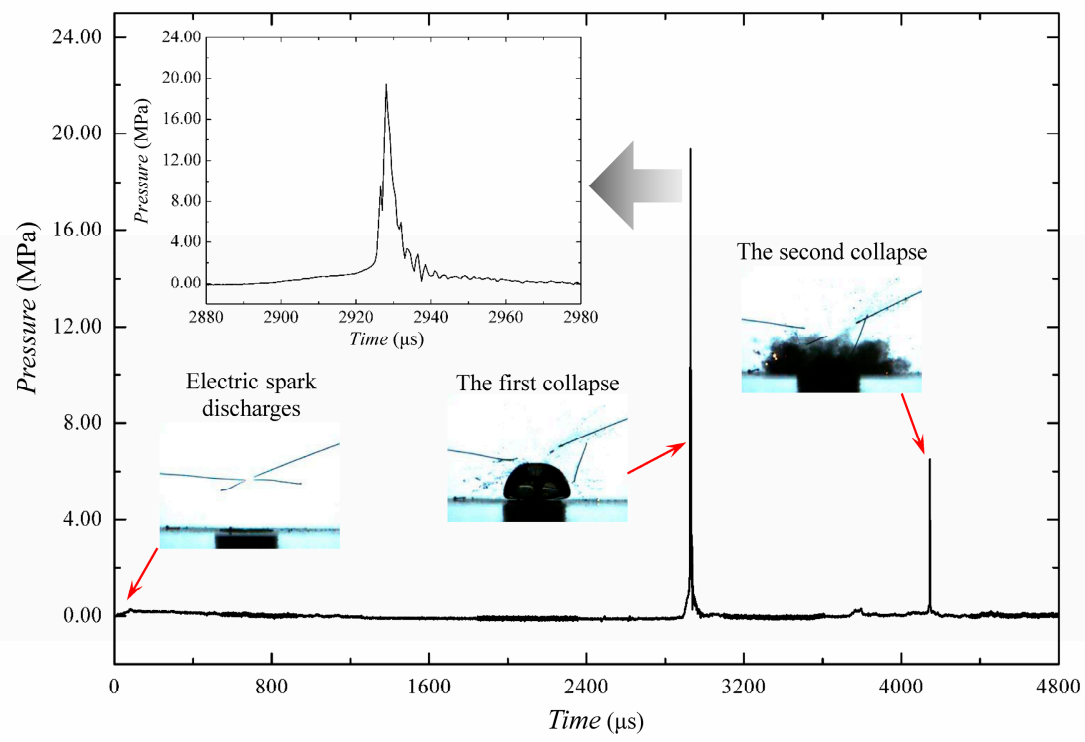

(a) $R_{\max }=9.98 \mathrm{~mm} ; \gamma=0.91$

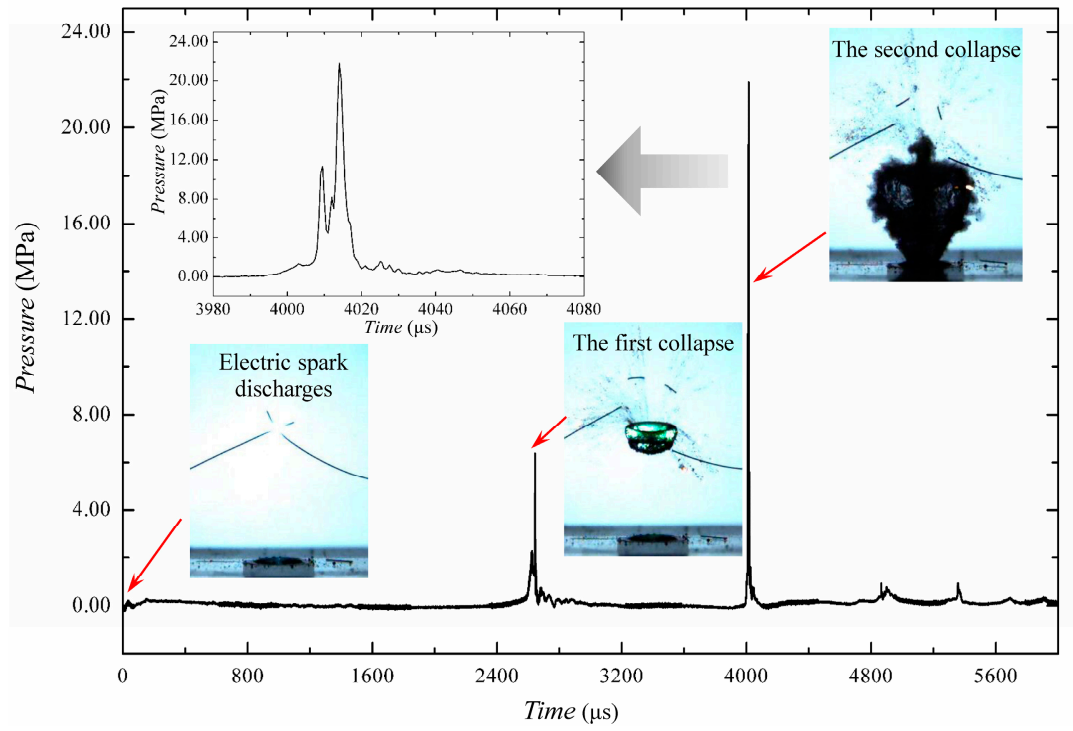

(b) $R_{\max }=11.02 \mathrm{~mm} ; \gamma=1.79$

Figure 4. The impulsive process generated by cavitation bubble collapse for (a) $\gamma=0.91 ;(\mathbf{b}) \gamma=1.79$.

The maximum radius of the cavitation bubble in Figure $4 \mathrm{~b}$ is $11.02 \mathrm{~mm}$, and the distance from the center to the wall surface is $19.73 \mathrm{~mm}$ when the cavitation bubble reaches the maximum radius. 
The first peak and the second peak are the impact on the wall surface during the first collapse and rebound collapse of the cavitation bubble, with the peak pressure values of $6.38 \mathrm{MPa}(2.64 \mathrm{~ms})$ and $21.89 \mathrm{MPa}(4.01 \mathrm{~ms})$, respectively. The minimum distance to the wall surface when the cavitation bubble shrinks to the minimum volume for the first time is $13.81 \mathrm{~mm}$, and at this moment, the dimensionless distance from the center of the cavitation bubble to the wall surface is 5.06 . The cavitation bubble will quickly move to the wall surface in the rebound evolution process; when the cavitation bubble contracts to the minimum volume once more, the cavitation bubble is close to the wall surface, which is as shown in Figure $4 b$.

It can be clearly seen from Figure 4 that when the dimensionless distance $\gamma$ is small, the impact of the first collapse of the cavitation bubble on the wall surface is greater than the impact during rebound collapse; when $\gamma$ can meet a certain condition and the cavitation bubble completes the impact of the first collapse on the wall surface, the rebound cavitation bubble will quickly move to the wall surface, and the impact pressure greater than that of the first collapse will act on the wall surface once more.

Shaw et al. [6] used laser-induced cavitation bubbles and a pressure (voltage) transducer to obtain the waveform of the impact of the first collapse of cavitation bubbles on the wall when $\gamma$ is between 0.56 and 1.5. The waveform of the impact of the first collapse of cavitation bubbles on the wall reported in the literature is very similar to that in Figure 4a. The similarities between the two are as follows: (1) there are three peaks in the pressure rise. Except the maximum peak, the other two are distributed on both sides of the maximum pressure value; (2) the time interval between the two peaks before the pressure in the experiment increases to the maximum value is $8 \mu \mathrm{s}$, and the time interval during the pressure drop is $3 \mu \mathrm{s}$; (3) the time of the pressure rise is slightly greater than that of the pressure drop. The differences between the two are as follows: (1) the time span between several peaks is different. The time span in the experiment in this paper is greater than the corresponding value in the literature; (2) the time span of the pressure rise and that of the pressure drop differ. The time of the pressure rise in this experiment is about $13 \mu \mathrm{s}$, and that of the pressure drop about $6 \mu \mathrm{s}$, which are both greater than that in the literature. The above phenomenon is mainly caused by the influence of the size of the cavitation bubbles. The maximum radius of the corresponding cavitation bubble is $1.17 \mathrm{~mm}$ when $\gamma$ is 0.89 in the literature, and $9.98 \mathrm{~mm}$ when $\gamma$ is 0.91 in this paper.

The impact of the jet on the wall will cause a water hammer effect. The water hammer pressure keeps a linear relation with the jet velocity and is calculated as follows [3]:

$$
P_{w h}=\frac{\rho_{w} c_{w} \rho_{s} \mathcal{C}_{s}}{\rho_{w} c_{w}+\rho_{s} c_{s}} v_{t}
$$

where $\rho_{w}$ and $c_{w}$ are the density and sound velocity of the jet medium, respectively; $\rho_{s}$ and $c_{S}$ are the density and sound velocity of the solid wall, respectively; and $v_{t}$ is the velocity of the jet as it impacts the wall. The wall used in this paper is made of plexiglass, with a density and sound velocity of $2700 \mathrm{~kg} / \mathrm{m}^{3}$ and $2692 \mathrm{~m} / \mathrm{s}$, respectively. The density and sound velocity of the jet medium are $1000 \mathrm{~kg} / \mathrm{m}^{3}$ and $1435 \mathrm{~m} / \mathrm{s}$, respectively.

Figure 5 is a high-speed photographic image of the collapse of cavitation bubbles in Figure $4 b$, where $v$ is the micro-jet velocity. The frame-rate used in the experiment was 150,000 frames per second, and the pictures in Figure 5 were acquired every 15 frames based on the experimental rate. The micro-jet was formed during the first collapse of the cavitation bubble when the velocity of the micro-jet was approximately $74.96 \mathrm{~m} / \mathrm{s}$. When the cavitation bubble entered the rebound stage, a micro-jet was again formed with impact on the wall. The micro-jet developed gradually and eventually imposed an impact on the wall, with its speed decreasing to approximately $13.00 \mathrm{~m} / \mathrm{s}$ when arriving at the wall. According to the formula for the water hammer pressure, the impact pressure of the micro-jet formed from the rebound cavitation bubbles against the wall is estimated to be $21.36 \mathrm{MPa}$, and the pressure peak measured by the pressure sensor is $21.89 \mathrm{MPa}$, both of which are very close. It can be seen that the impact of the micro-jet formed from the rebound cavitation bubbles against the wall mainly comes from the impact of the micro-jet. For the impact of the micro-jet from the first collapse 
on the wall when the distance between the bubble and the wall is small, due to the limited speed of the high-speed camera used in this experiment, no clear shock wave can be obtained. Therefore, it is currently impossible to tell whether the main reason for the pressure peak of the impact of the cavitation bubble on the wall from the first collapse is the shock wave or the micro-jet.

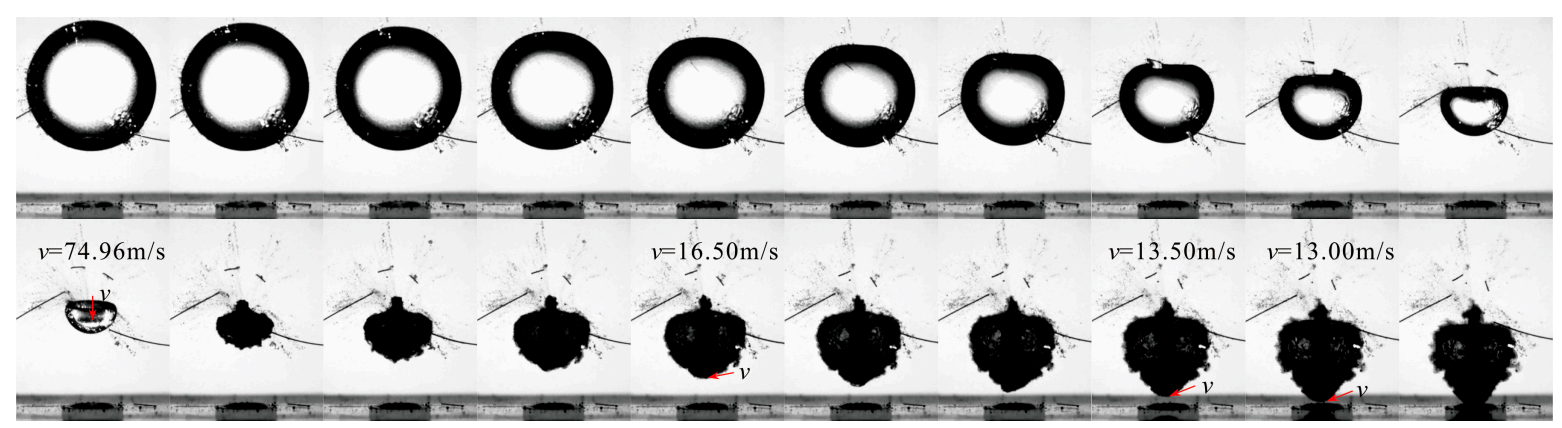

Figure 5. Process of the impact of micro-jet of rebound cavitation bubbles on a wall (frame-rate: 150,000 fps; exposure time: $\left.5.06 \mu \mathrm{s} ; R_{\max }=11.02 \mathrm{~mm}, \gamma=1.79\right)$.

\subsection{Influence of the Distance Between the Cavitation Bubble and Wall on the Impact Strength of the Wall Surface}

In the whole process of cavitation bubble evolution, when the dimensionless distance $\gamma$ meets a certain condition, the second collapse impact strength on the wall surface is greater than the first collapse impact strength. Thus, in this section, the dimensionless distance $\gamma$ and the impact strength on the wall surface are systematically studied to gain the relationship of the peak impact pressure on the wall surface at cavitation bubble collapse stages under different $\gamma$ conditions.

In order to obtain the evolution process and impact process of the cavitation bubble with different dimensionless distances $\gamma$ on the wall surface, the distance from the discharge electrode to the wall surface (with pressure sensor buried inside) will be slightly adjusted in the testing, and the characteristic radius $R_{\text {max }}$ of the cavitation bubble will be changed under each $h$ condition. For the peak value of the impact strength of the cavitation bubble on the wall surface, the maximum pressure of the first impact and second impact during the impact of the cavitation bubble on the wall surface is selected in this section to reflect the impact strength of the cavitation bubble on the wall surface.

Figure 6 shows the relationship between the dimensionless distance $\gamma$ and the wall peak pressure; therein, the horizontal axis indicates the dimensionless distance $\gamma$ and the vertical axis indicates the corresponding pressure peaks on the wall surface during the first and second collapses of the cavitation bubble. It can be seen from Figure 6 that for the first collapse of the cavitation bubble, the relationship between the dimensionless distance $\gamma$ and pressure peak on the wall surface shows an exponential-type distribution overall: with the gradual decrease of $\gamma$, the maximum value of the impact on the wall surface rapidly increases. Then, the peak value will increase first and then rapidly decrease as a whole during the impact of the second collapse of the cavitation bubble on the wall surface. When the distance between the cavitation bubble and the wall increases, the peak pressure on the wall can be divided into the following three parts: when $\gamma$ is less than 1.33 , the impact of the first collapse of the cavitation bubble on the wall surface is greater than the impact of the second collapse on the wall surface; when $\gamma$ between the cavitation bubble and wall is greater than 1.33 and less than 2.37 , the impact strength on the wall surface caused by the second collapse of the cavitation bubble will be greater than the impact strength of the first collapse on the wall surface; when $\gamma$ is greater than 2.37, the impact of the first collapse of the cavitation bubble on the wall surface is greater than the impact of the second collapse on the wall surface, and the maximum of the first impact peak is less than $4 \mathrm{MPa}$. 


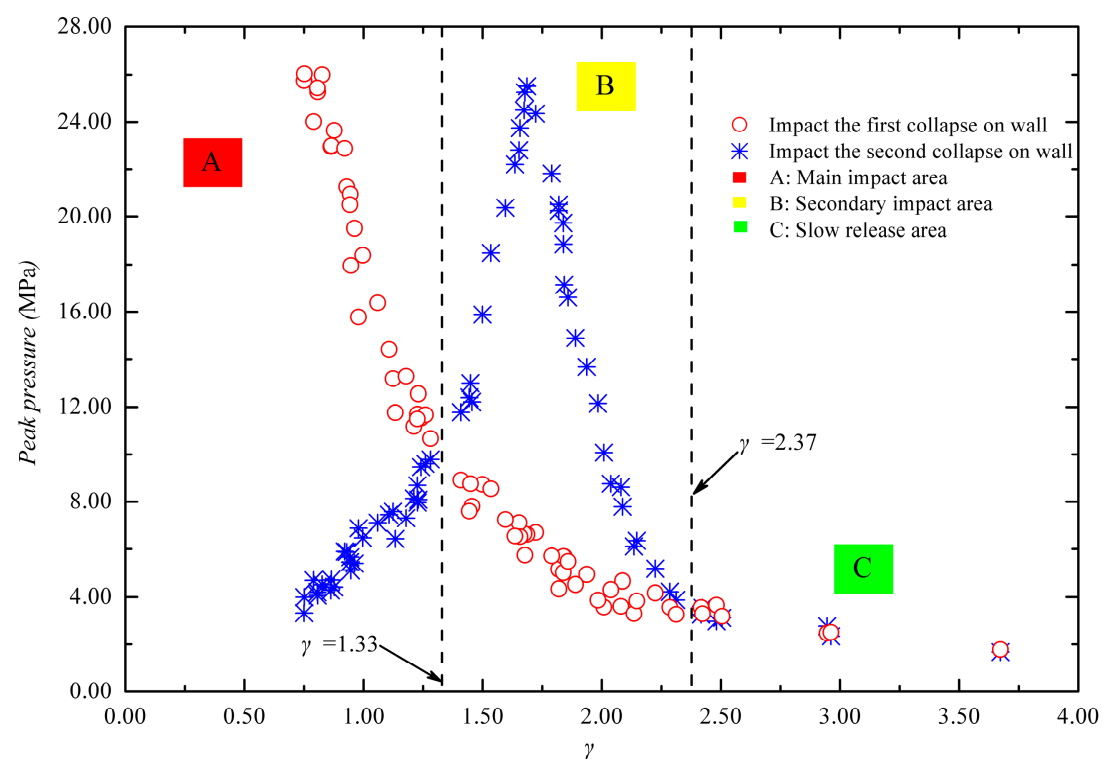

Figure 6. The relationship between the dimensionless distance $(\gamma)$ and the wall peak pressure.

It can be seen from the above analysis that for the collapse of cavitation bubbles near the wall surface, when the dimensionless distance $\gamma$ is less than 2.37 , the impact of the first and second collapses of the cavitation bubble on the wall surface is huge; when $\gamma$ is greater than 1.33 and less than 2.37, the impact on the wall surface caused due to the second collapse of the cavitation bubble is greater than the impact on the wall surface caused due to the first collapse. The reason why the above impact characteristics are seen can be analyzed from the following two aspects: on the one hand, when the bubble is very close to the wall surface, during the first bubble-asymmetric shrinkage, a micro-jet with a direct impact on the wall is formed. When the cavitation bubble is a little further away from the wall surface, the impact of the wall on the first shrinkage form of the cavitation bubble is weakened, and a micro-jet is formed during the shrinkage of the cavitation bubble. The micro-jet towards the wall surface drives the cavitation bubble, and finally, during the second shrinkage, the micro-jet is formed. This micro-jet can impose an impact on the wall. Although the energy of the cavitation bubble itself is attenuated after the first cycle of evolution, the impact of the micro-jet from the second shrinkage on the wall is still considerably large. As the distance between the cavitation bubble and the wall surface further increases, the bubble during the second shrinkage obviously moves toward the wall surface, but the resulting micro-jet from the second collapse is not sufficient to impose an impact on the wall, and then the bubble enters the next expansion and contraction stage. On the other hand, the cavitation bubble has a certain energy after the birth. The energy is dissipated due to the viscosity of the water body and other factors after the first and second expansion. After the two-fold expansion and shrinkage, the energy is significantly reduced. As a result, less obvious movement toward the wall is observed and the impact on the wall is reduced greatly in the subsequent evolution of the cavitation bubble.

Philipp and Lauterborn [3] studied the deterioration of the wall under different bubble-wall conditions when the cavitation bubble's $R_{\max }=1.45 \mathrm{~mm}$ with the laser-induced cavitation technology. There were over 100 groups of samples. By analyzing the deterioration and bubble-wall distance $\gamma$, it is found that when $\gamma \geq 2.2$, no deterioration was found on the solid wall; when $2.2 \geq \gamma \geq 1.5$, the area of deterioration increased with the decrease of $\gamma$. By comparing the deterioration in the literature and the impact of the cavitation bubble on the wall obtained in this paper, it can be found that the critical values are basically consistent. Since a low-pressure discharge-induced cavitation bubble is used here, it is more difficult to achieve cavitation under the condition of smaller values of $\gamma$. Furthermore, the cavitation bubble was larger with the low-voltage underwater discharge technology used in this paper, and the size in the literature is smaller, which may cause the slight difference in the 
critical value between the paper and the literature. This difference, however, does not affect the zoning characteristics of the impact of the cavitation bubble on the wall.

\section{Conclusions}

Through the combination of the high-speed dynamic collection and analysis system and transient pressure testing system, the impact characteristics of the collapse of the low-voltage spark-discharge cavitation bubble on the wall surface are studied in this paper, drawing the following important conclusions:

(1) The dimensionless distance between the cavitation bubble and wall $(\gamma)$ has an important influence on the formation of the micro-jet in the late evolution stage of the cavitation bubble. When the dimensionless distance $\gamma$ is less than 1.33, a micro-jet directly impacting the wall surface can be formed during the first contraction of the cavitation bubble; subsequently, the collapse of the main impact area will occur. When $\gamma$ is within 1.33 2.37, the second collapse of the cavitation bubble after rebound will cause a greater impact on the wall surface, then the collapse of the secondary impact area will occur. When $\gamma$ is greater than 2.37, the influence of wall on the development of a micro-jet is not obvious, that is, collapse in the slow release area occurs.

(2) Concerning the peak value of the impact pressure of the cavitation bubble collapse on the wall surface, the impact of cavitation bubbles on the wall surface during the main impact gradually decreases with the increase of the distance between the cavitation bubble and the wall, and the impact of the cavitation bubble on the wall surface during the secondary impact increases first and then decreases. More specifically, when the dimensionless distance $\gamma$ is less than 1.33 , the impact on the wall surface due to cavitation bubble collapse is mainly from the first collapse, and with increasing $\gamma$, the impact of the second collapse on the wall surface increases. When $\gamma$ is within 1.33 2.37, the impact of the cavitation bubble on the wall surface during the first collapse will be less than the impact strength of the re-collapse after rebound on the wall surface with increase of $\gamma$. When $\gamma$ is greater than 2.37, the impact of the first and second collapses of the cavitation bubble on the wall surface will decrease considerably.

The above conclusions have potential theoretical value for the anti-erosion design of hydraulic engineering, the erosion resistance of blades in hydraulic machinery and the better exertion of the cavitation effect in ultrasonic cleaning technology.

Author Contributions: Conceptualization, W.X. and J.L.; Methodology, J.D. and J.L.; Software, J.L.; Validation, W.X. and J.D.; Formal Analysis, J.L.; Investigation, J.L.; Resources, J.L.; Data Curation, Y.Z. and Q.Z.; Writing-Original Draft Preparation, J.L.; Writing-Review \& Editing, J.D.; Visualization, J.L.; Supervision, W.X.; Project Administration, W.X.; Funding Acquisition, J.D.

Funding: This research was funded by the National Key R\&D Program of China (Grant No.2016YFC0401901) and the National Natural Science Foundation of China (Grant No.51409180).

Conflicts of Interest: The authors declare no conflict of interest.

\section{References}

1. Shima, A.; Takayama, K.; Tomita, Y.; Ohsawa, N. Mechanism of impact pressure generation from spark-generated bubble collapse near a wall. Aiaa J. 1983, 21, 55-59. [CrossRef]

2. Tomita, Y.; Shima, A. Mechanisms of impulsive pressure generation and damage pit formation by bubble collapse. J. Fluid Mech. 1986, 169, 535-564. [CrossRef]

3. Philipp, A.; Lauterborn, W. Cavitation erosion by single laser-produced bubbles. J. Fluid Mech. 1998, 361, 75-116. [CrossRef]

4. Mitelea, I.; Bordeaşu, I.; Pelle, M.; Crăciunescu, C. Ultrasonic cavitation erosion of nodular cast iron with ferrite-pearlite microstructure. Ultrason. Sonochem. 2015, 23, 385-390. [CrossRef] [PubMed]

5. Dong, F.; Li, X.; Zhang, L.; Ma, L.; Li, R. Cavitation erosion mechanism of titanium alloy radiation rods in aluminum melt. Ultrason. Sonochem. 2016, 31, 150-156. [CrossRef] [PubMed] 
6. Shaw, S.J.; Schiffers, W.P.; Emmony, D.C. Experimental observations of the stress experienced by a solid surface when a laser-created bubble oscillates in its vicinity. J. Acoust. Soc. Am. 2001, 110, 1822. [CrossRef] [PubMed]

7. Lindau, O.; Lauterborn, W. Cinematographic observation of the collapse and rebound of a laser-produced cavitation bubble near a wall. J. Fluid Mech. 2003, 479, 327-348. [CrossRef]

8. Liu, X.M.; He, J.; Lu, J.; Ni, X.W. Effect of surface tension on a liquid-jet produced by the collapse of a laser-induced bubble against a rigid boundary. Opt. Laser Technol. 2009, 41, 21-24. [CrossRef]

9. Xu, R.; Zhao, R.; Cui, Y.; Lu, J.; Ni, X. The collapse and rebound of gas-vapor cavity on metal surface. Opt. Int . J. Light Electron. Opt. 2009, 120, 115-120. [CrossRef]

10. Ren, X.D.; He, H.; Tong, Y.Q.; Ren, Y.P.; Yuan, S.Q.; Liu, R.; Zou, C.Y.; Wu, K.; Sui, S.; Wang, D.S. Experimental investigation on dynamic characteristics and strengthening mechanism of laser-induced cavitation bubbles. Ultrason. Sonochem. 2016, 32, 218-223. [CrossRef] [PubMed]

11. Rochester, M.C.; Brunton, J.H. Surface pressure distribution during drop impingement. In Proceedings of the Fourth International Conference on Rain Erosion and Related Phenomena, Neues Schloss, Meersburg, Germany, 8-10 May 1974; pp. 371-393.

12. Klaseboer, E.; Fong, S.W.; Turangan, C.K.; Khoo, B.C.; Szeri, A.J.; Calvisi, M.L.; Sankin, G.N.; Zhong, P. Interaction of lithotripter shockwaves with single inertial cavitation bubbles. J. Fluid Mech. 2007, 593, 33-56. [CrossRef] [PubMed]

13. Lauterborn, S.; Urban, W. Ultrasonic cleaning of submerged membranes for drinking water applications. J. Acoust. Soc. Am. 2008, 123, 3291. [CrossRef]

14. Verhaagen, B.; Fernández, R.D. Measuring cavitation and its cleaning effect. Ultrason. Sonochem. 2016, 29, 619-628. [CrossRef] [PubMed]

15. Fong, S.W.; Adhikari, D.; Klaseboer, E.; Khoo, B.C. Interactions of multiple spark-generated bubbles with phase differences. Exp. Fluids 2009, 46, 705-724. [CrossRef]

16. Pain, A.; Terence Goh, B.H.; Klaseboer, E.; Ohl, S.W.; Cheong Khoo, B. Jets in quiescent bubbles caused by a nearby oscillating bubble. J. Appl. Phys. 2012, 111, 137. [CrossRef]

17. Luo, J.; Xu, W.L.; Niu, Z.P.; Luo, S.J.; Zheng, Q.W. Experimental study of the interaction between the spark-induced cavitation bubble and the air bubble. J. Hydrodyn. 2013, 25, 895-902. [CrossRef]

18. Luo, J.; Xu, W.L.; Li, R. High-speed photographic observation of collapse of two cavitation bubbles. Sci. Chin. Technol. Sci. 2016, 59, 1707-1716. [CrossRef]

19. Goh, B.H.T.; Ohl, S.W.; Klaseboer, E.; Khoo, B.C. Jet orientation of a collapsing bubble near a solid wall with an attached air bubble. Phys. Fluids 2014, 26, 221-240. [CrossRef]

20. Xu, W.L.; Zhang, Y.L.; Luo, J.; Arong; Zhang, Q.; Zhai, Y.W. The impact of particles on the collapse characteristics of cavitation bubbles. Ocean Eng. 2017, 131, 15-24. [CrossRef]

21. Bing, H.T.G.; Shi, W.G.; Ohl, S.W.; Khoo, B.C. Spark-generated bubble near an elastic sphere. Int. J. Multiphase Flow 2016, 90, 156-166. [CrossRef]

22. Cui, P.; Zhang, A.M.; Wang, S.; Khoo, B.C. Ice breaking by a collapsing bubble. J. Fluid Mech. 2018, 841, 287-309. [CrossRef]

23. Duncan, J.H.; Zhang, S. On the interaction of a collapsing cavity and a compliant wall. J. Fluid Mech. 1991, 226, 401-423. [CrossRef]

24. Chahine, G.L.; Kalumuck, K.M.; Duraiswami, R. Bubble dynamics fluid-structure interaction simulation by coupling fluid BEM and structural FEM codes. Int. J. Multiphase Flow 1995, 9, 861-883. [CrossRef]

25. Chahine, G.L.; Kalumuck, K.M.; Hsiao, C.T. Simulation of surface piercing body coupled response to underwater bubble dynamics utilizing 3dynafs, a three-dimensional bem code. Comput. Mech. 2003, 32, 319-326. [CrossRef]

26. Zhang, Y.L.; Yeo, K.S.; Khoo, B.C.; Wang, C. 3d jet impact and toroidal bubbles. J. Comput. Phys. 2001, 166, 336-360. [CrossRef]

27. Wang, C.; Khoo, B.C. An indirect boundary element method for three-dimensional explosion bubbles. J. Comput. Phys. 2004, 194, 451-480. [CrossRef]

28. Best, J.P. The formation of toroidal bubbles upon the collapse of transient cavities. J. Fluid Mech. 1993, 251, 79-107. [CrossRef] 
29. Klaseboer, E.; Hung, K.C.; Wang, C.; Wang, C.W.; Khoo, B.C.; Boyce, P.; Debono, S.; Charlier, H. Experimental and numerical investigation of the dynamics of an underwater explosion bubble near a resilient/rigid structure. J. Fluid Mech. 2005, 537, 387-413. [CrossRef]

30. Goh, B.H.; Oh, Y.D.; Klaseboer, E.; Ohl, S.W.; Khoo, B.C. A low-voltage spark-discharge method for generation of consistent oscillating bubbles. Rev. Sci. Instrum. 2013, 84, 014705. [CrossRef] [PubMed] 